\title{
Centennial climate change: The unknown variability zone
}

Michel Crucifix ${ }^{1}$ A. de Vernal ${ }^{2}$ and C. Franzke ${ }^{3}$

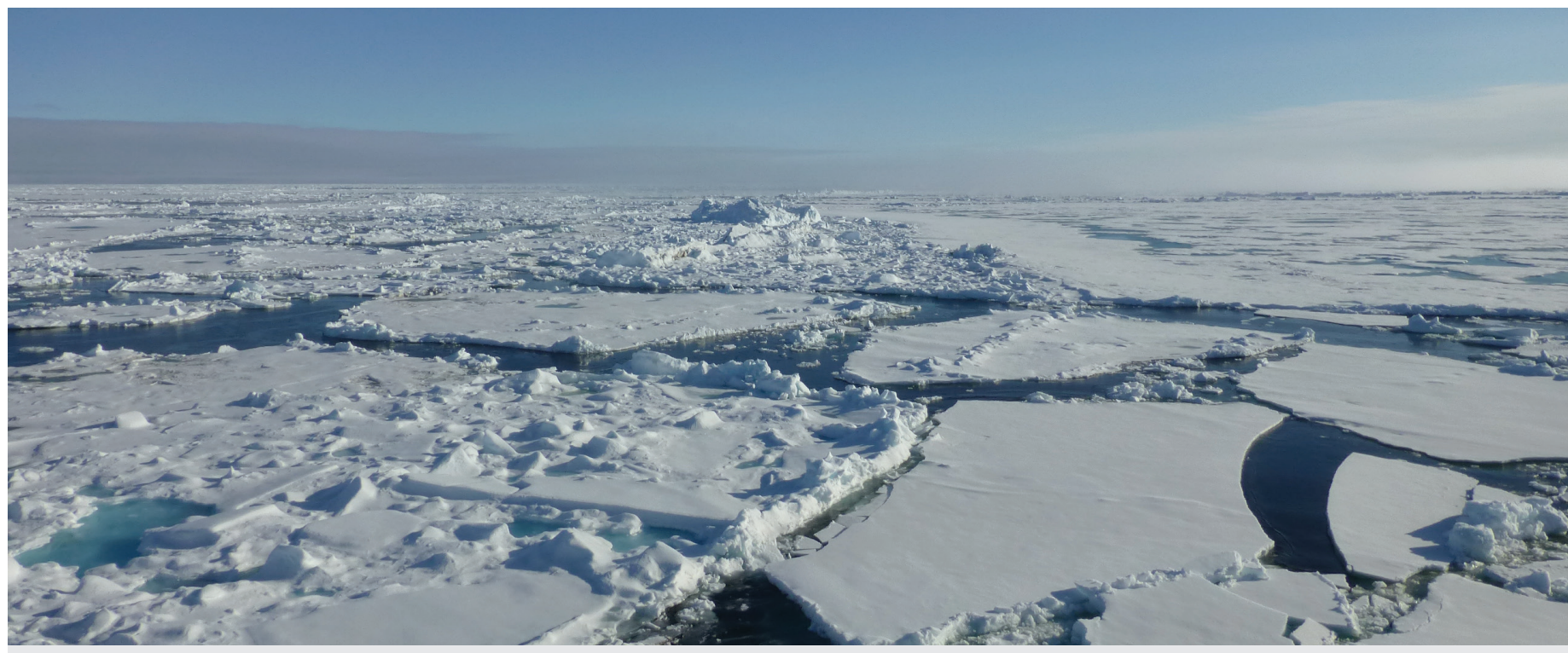

Power spectra of paleoclimate records show that climate variability exists at the centennial timescale. There is no variability gap, as previously thought. Photo credit: Anne de Vernal.

Arguably, centennial variability has been the forgotten orphan of climate dynamics theory. In a 1990 paper titled "Three basic problems of palaeoclimate modelling", the late Barry Saltzman presented the centennial timescale as a zone with a variability gap - a demilitarized zone, separating people interested in weather from those interested in the slow evolution of climate.

Saltzman was putting forward an idea that has, until recently, deeply penetrated our paradigms of climate modeling. General circulation models generate weather. A socalled "climate snapshot" is often an average of the generated weather over a few centuries or so. At the other extreme, the dynamics of ice ages are simulated with models which do not include weather variability. We know today that this separation is no longer tenable. There is centennial variability, which seamlessly connects weather to the great ice ages.

The study of centennial variability is thus important for gaining a complete understanding of climate variability. It is important because a deep knowledge of the causes and the amplitude of climate variations at the centennial timescale is needed to properly put anthropogenic climate change and future climate projections into context. Beyond decadal mean temperature, we are concerned about trends and changes in the occurrence of extreme events, changes in ocean circulation and changes in quasioscillatory regimes such as ENSO.

All of this may occur at the centennial scale. But studying centennial variability is also important for those who are interested in the slowest timescales, as centennial dynamics may influence the timing and speed of phenomena such as glacial inceptions and deglaciations. For example, sea-level fluctuations during interglacial periods are now known to have been larger than previously believed.

We are thus left with the difficult task of quantifying the amplitude of centennial variability and to understand its causes. Some of the challenges bear on the experts in the collection and analysis of paleoclimate data. Examples taken from marine records, sea ice and tree rings highlight the unfinished path still to accomplish to separate climate from non-climate variability in paleoclimate records. We should thus be wary of naive and superficial interpretations of power spectra. On the other hand, explanations for the causes of centennial variability generate their share of heated debates.

As we know, the climate system is complex enough to generate its own internal oscillations, like the El-Niño Southern Oscillations or Dansgaard-Oeschger events. There is no reason not to have such modes in the centennial band. Dijkstra and Von der Heydt (p. 150) provide one such example.

Besides, variability emerging from the chaos of atmospheric and oceanic motion may also propagate upwards and downwards throughout the frequency spectrum to produce what we know as the background spectrum. There is a controversy as to whether the background is mainly generated by linear processes of accumulation and relaxation of fluctuations, or whether it involves non-linear dynamics at a deep level, akin to turbulent processes, which are by nature intermittent and "spiky". A wise and well-informed use of statistical modeling will be helpful to instruct the debate.

The PAGES working group "Climate variability Across Scales (CVAS)" has been established for these reasons, and brings together experts in time series analysis and nonlinear geophysics with paleoclimate and data scientists.

Finally, we need to pay more attention to the external forcing of climate change. We still know little about the centennial variability of solar forcing or about possible changes in volcanic activity at this timescale. Ice-age modelers are, however, too familiar with the idea that even weak external forcing may combine with internal dynamics to generate large amplitude response.

With the urgency of anthropogenic climate change, we have to do everything possible to make sure we have a good grasp on centennial dynamics. We are not there yet. However, we hope that this special issue will more widely spread discussions about this topic and increase interest in centennial variability.

\section{AFFILIATIONS}

'Earth and Life Institute, Université catholique de Louvain, Louvain-la-Neuve, Belgium

${ }^{2}$ GEOTOP, Université du Québec à Montréal, Canada ${ }^{3}$ Meteorological Institute and Center for Earth System Research and Sustainability, University of Hamburg, Germany

CONTACT

Michel Crucifix: michel.crucifix@uclouvain.be 